\title{
To Reduce the Hemocytometer Measurement Uncertainty of White Blood Cell Count by the Double Staining of Cell Nucleus Ying-Ying LIU $^{\mathrm{a}, *}$, Jing WANG
}

\author{
National Institute of Metrology, Beijing, P. R. China Beijing, 100029 \\ aemail: liuyy@nim.ac.cn \\ ${ }^{*}$ Corresponding author
}

Keywords: Hemocytometer, Measurement uncertainty, White blood cell.

\begin{abstract}
Acridine orange (AO) and ethidium bromide (EB) are two fluorescent dyes for cell nuclei staining. The nucleus images by these two staining can make it easy to differentiate white blood cell (WBC) from red blood cell (RBC). We applied this principle to the white blood cell count by hemocytometer and this can eliminate the errors caused by misjudging of WBC. At the same time, microscopic imaging can improve the accuracy of the volume measurement. The extended relative uncertainty of two measurements have been analyzed and compared.
\end{abstract}

\section{Introduction}

Blood cell analysis is one of the most commonly used laboratory testing indicators, the accuracy of the results directly affect the diagnosis and treatment of patients [1]. Although various types of automatic blood cell analyzers have been widely used in clinical testing, the blood cell count by microscope is still the most reliable and classical counting technique, and the absolute measurement method of the particle count [2]. However, the subjective error caused by the manual reading is an important source of the influence of the uncertainty [3]. The number of RBC in the blood is thousands of times of the number of WBC. The error caused by the red blood cells to be mistaken as white blood cells will reduce the measurement accuracy. With the development of fluorescence labeling and micro imaging technology, cell shape can be more accurately determined which has greatly expanded the observation angle and the application field of micro technology. Therefore this study using nuclear DNA specific dye (acridine orange / ethidium bromide) effectively distinguish leukocytes with nuclear and red nucleus cells without nuclear and improve the accuracy of WBC count by hemocytometer counts.

\section{Materials and Methods}

\section{Instruments and Reagents}

The main instruments are the fluorescence microscope (Zeiss, Imager M2) and blood cell counting plate (Qiujing Shanghai).AO/EB double staining kit was obtained from Solarbio Science and Technology Co. Ltd. 


\section{White Blood Cell Staining}

Acridine orange solution and ethidium bromide were prepared as working solution according to kit's instructions. The blood cells were stained as 90 microliters of sample with acridine orange solution $(5 \mu \mathrm{L})$ and ethidium bromide solution $(5 \mu \mathrm{L})$, incubated at room temperature to avoid light for 15 minutes.

\section{Imaging Condition and Data Analysis}

The green fluorescence channel (excitation wavelength: 490nm 510nm and emission wavelength: $520 \mathrm{~nm} \sim 550 \mathrm{~nm}$ ) and the red fluorescence channel (excitation wavelength: 540nm 552nm and emission wavelength: 590nm infinity) were used. The microscopic fluorescence images were overlayed and analyzed by the Vision Rel. Axio 4.7.

\section{Results and Discussion}

According to the requirements of white blood cell measurement in National Guide to Clinical Laboratory Procedures (3rd Edition) [4], the red blood cell lysis and sample dilution were carried out. According to the JJF1059-1999 Evaluation and Expression of Uncertainty in Measurement [5], the white blood cell measurement model was set up as follows: $\mathrm{C}=\mathrm{F}^{*} \mathrm{~N} / \mathrm{V}$.

Where $\mathrm{C}$ - the sample cell concentration; F- dilution factor; $\mathrm{N}$ - cell count of the blood cell counter; $\mathrm{V}$ - the measurement volume of the blood cell counter plate.

In order to detect the measurement repeatability of the double staining method, the study was carried out by three independent operators. The total 10 separate samples were measured by the conventional hemocytometer and our method. The results showed: relative standard deviation (RSD) of the conventional method would be $4.37 \%$ and RSD of the new one would be $2.53 \%$.

\section{Measurement Uncertainty Analysis for Conventional Hemocytometer Method}

were analyzed, including the following aspects:

(1) The repeatability and reproducibility of measurement: according to the experimental data mentioned above, the uncertainty can be: $u_{\text {rel }}(A)=R S D / \sqrt{n-1}=0.0437 / 3=0.0146$

(2) Dilution: in order to reach the appropriate concentration range of the blood cell, the clinical blood sample is required for a dilution, and the steps were: transferred $5 \mathrm{~mL}$ fresh blood to $100 \mathrm{~mL}$ volume flask by $5 \mathrm{~mL}$ pipette, and then added the PBS buffer (pH7.2, 0.1M) to the tick mark.

The permissible deviation of $5 \mathrm{~mL}$ pipette (class A) cannot be over $\pm 0.025 \mathrm{~mL}$, which is in accordance with the triangular distribution. So the volume uncertainty of $5 \mathrm{~mL}$ pipette would be calculated as $0.025 / \sqrt{6}=0.01 \mathrm{~mL}$, Bending surface reading can introduce related uncertainty, and the inner diameter is $2 \mathrm{~mm}$, the meniscus position error would be $1.0 \mathrm{~mm}$, so the absolute error would be $3.15 \mu \mathrm{L}$. The reading error is in the triangular distribution, and the standard of the $5 \mathrm{~mL}$ shift is as follows: 
$0.00315 / \sqrt{6}=0.0013 \mathrm{ml}$. Therefore, the combined uncertainty of the $5 \mathrm{~mL} 5 \mathrm{~mL}$ pipette should be: $u\left(V_{1}\right)=\sqrt{(0.01)^{2}+(0.0013)^{2}}=0.01$.

The permissible deviation of $100 \mathrm{~mL}$ volume flask (class A) cannot be over $\pm 0.1 \mathrm{~mL}$, which is in accordance with the triangular distribution. So the volume uncertainty would be calculated as $0.1 / \sqrt{6}=0.041 \mathrm{~mL}$. Bending surface reading can introduce related uncertainty, and the inner diameter is $10 \mathrm{~mm}$, the meniscus position error would be $1.0 \mathrm{~mm}$, so the absolute error would be $78.5 \mu \mathrm{L}$. The reading error is in the triangular distribution, and the standard of the $5 \mathrm{~mL}$ shift is as follows: $0.0785 / \sqrt{6}=0.0032 \mathrm{ml}$. Therefore, the combined uncertainty of the $5 \mathrm{~mL} 5 \mathrm{~mL}$ pipette should be: $u\left(V_{2}\right)=\sqrt{(0.041)^{2}+(0.032)^{2}}=0.052 \mathrm{~mL}$.

(3) Measuring volume: America National Bureau of standards (NBS) provides generous error for each grid side should be less than $1 \%$, the depth of the error should be less than $2 \%$. The volume relative uncertainty is estimated by triangular distribution as:

$u_{r e l}\left(V_{m}\right)=\sqrt{\left(\frac{S_{x}}{\sqrt{6}}\right)^{2}+\left(\frac{S_{y}}{\sqrt{6}}\right)^{2}+\left(\frac{S_{z}}{\sqrt{6}}\right)^{2}}=\sqrt{\left(\frac{0.01}{\sqrt{6}}\right)^{2}+\left(\frac{0.01}{\sqrt{6}}\right)^{2}+\left(\frac{0.02}{\sqrt{6}}\right)^{2}}=0.01$

According to the mathematical model, the measurement uncertainty of WBC count could be determined as:

$u_{r e l}(C)=\sqrt{u_{r e l}^{2}(A)+\left[\frac{u\left(V_{1}\right)}{V_{1}}\right]^{2}+\left[\frac{u\left(V_{2}\right)}{V_{2}}\right]^{2}+u_{r e l}^{2}\left(V_{m}\right)}=\sqrt{(0.0146)^{2}+\left(\frac{0.01}{5}\right)^{2}+\left(\frac{0.052}{100}\right)^{2}+(0.01)^{2}}$

$=0.0178$

\section{Measurement Uncertainty Analysis for New Method}

The new fluorescence double staining microscopy image method is used for the white blood cell count, and the method is similar to the conventional method. The difference lies in:

(1) The repeatability and reproducibility of measurement have been decreased, so its measurement uncertainty has been reduced accordingly.

$u_{r e l}(A)=R S D / \sqrt{n-1}=0.0253 / 3=0.0084$

(2) Measuring volume, due to the size uncertainty of microscopic image pixel dimensions was significantly less than that in hemocytometer itself. Through the analysis of the pixel size of the micro image, the expanded uncertainty of the $\mathrm{X}$ axis and the $\mathrm{Y}$ axis of each pixel is obtained with the $\mathrm{x}=0.627 \pm 0.00096 \mu \mathrm{m}, \mathrm{y}=0.625 \pm$ $0.00096 \mu \mathrm{m}$ and the relative uncertainty is significantly smaller than the $\mathrm{X}$ axis and the $\mathrm{Y}$ axis length of hemocytometer: 
$u_{r e l}\left(V_{m}\right)=\sqrt{u_{r e l}^{2}(\mathrm{X})+u_{r e l}^{2}(\mathrm{Y})+\left(\frac{S_{z}}{\sqrt{6}}\right)^{2}}=\sqrt{(0.000766)^{2}+(0.000768)^{2}+\left(\frac{0.02}{\sqrt{6}}\right)^{2}}$

$=0.0082$

Therefore, the combined measurement relative uncertainty of the white blood cell count by the double staining method was calculated as:

$$
\begin{aligned}
& u_{r e l}(C)=\sqrt{u^{2}{ }_{r e l}(A)+\left[\frac{u\left(V_{1}\right)}{V_{1}}\right]^{2}+\left[\frac{u\left(V_{2}\right)}{V_{2}}\right]^{2}+u^{2}{ }_{r e l}\left(V_{m}\right)} \\
& =\sqrt{(0.0084)^{2}+\left(\frac{0.01}{5}\right)^{2}+\left(\frac{0.052}{100}\right)^{2}+(0.0082)^{2}} \\
& =0.0119
\end{aligned}
$$

Therefore, the expanded measurement relative uncertainty of the two methods could be:

\begin{tabular}{|l|l|l|}
\hline & Conventional & New \\
\hline The combined relative uncertainty & $1.78 \%$ & $1.19 \%$ \\
\hline The extended relative uncertainty & $3.66 \%$ & $2.38 \%$ \\
\hline
\end{tabular}

\section{Summary}

The nucleus fluorescence staining can make it easy to differentiate WBC from RBC, and can eliminate the errors caused by misjudging of WBC accordingly. At the same time, microscopic imaging can improve the accuracy of the volume measurement. The extended relative uncertainty of WBC measurements can be reduced, from $3.66 \%$ to $2.38 \%$.

\section{References}

[1] C. Yulong, Y. Jiaxin. Recent Development of hematology analysis technique and its clinical applies, Chin J Lab Med . 30 (2007) 365-370.

[2] Z. Wenge, L. Junjie. Study of blood cell counting certified reference materials.C Powder Sci Tech. 15(2009): 59-63.

[3] F. Ruhui, D. Weimin, S. Mingzhe. Analysis on Multi-factors Influencing Hematology Count. Prac J Cancer. 16(2001): 87.

[4] Y. Yingwu: National Guide to Clinical Laboratory Procedures, Third ed., Southeast University Press, Nanjing, 2006.

[5] L. Shenan, S. Changyan, L. Feng. JJF1059-1999 Evaluation and Expression of Uncertainty in Measurement. General Administration of Quality Supervision, Inspection and Quarantine of the People's Republic of China. 